\title{
Highly localized divergence within supergenes in Atlantic cod (Gadus morhua) within the Gulf of Maine
}

\author{
Bryan T. Barney ${ }^{1 *}$, Christiane Munkholm², David R. Walt ${ }^{2}$ and Stephen R. Palumbi ${ }^{1}$
}

\begin{abstract}
Background: Atlantic cod (Gadus morhua), is known to vary genetically across the North Atlantic, Greenland, and Newfoundland. This genetic variation occurs both spatially and temporally through decades of heavy fishing, and is concentrated in three linkage disequilibrium blocks, previously defined by pedigreed linkage mapping analysis. Variation within these genomic regions is correlated with both seawater temperature and behavioral ecotype. The full extent and nature of these linkage groups is important information for interpreting cod genetic structure as a tool for future fisheries management.

Results: We conducted whole genome sequencing for 31 individual cod from three sub-populations in the Gulf of Maine. Across the genome, we found 3,390,654 intermediate to high frequency Single Nucleotide Polymorphisms (SNPs). We show that pairwise linkage analysis among these SNPs is a powerful tool to detect linkage disequilibrium clusters by recovering the three previously detected linkage groups and identifying the 1031 genes contained therein. Across these genes, we found significant population differentiation among spawning groups in the Gulf of Maine and between Georges Bank and Gulf of Maine. Coordinated divergence among these genes and their differentiation at both short and long spatial scales suggests that they are acting as linked supergenes in local adaptation of cod populations.

Conclusions: Differentiation between SNPs in linkage disequilibrium blocks is the major signal of genetic differentiation between all groups tested within the Gulf of Maine. Our data provide a map of genes contained in these blocks, allowing an enhanced search for neutral genetic structure for demographic inference and fisheries modeling. Patterns of selection and the history of populations may be possible to identify in cod using this description of linkage disequilibrium blocks and future data sets to robustly separate neutral and selected genetic markers.
\end{abstract}

Keywords: Atlantic cod, Gadus morhua, Linkage disequilibrium, Linkage map, Genomic divergence, Supergene, Gulf of Maine

\section{Background}

Genomic islands of divergence are the result of selection on regions within the genome undergoing adaptive divergence between subgroups within a species $[1,2]$. These islands can manifest as regions of strong linkage disequilibrium due to genetic hitchhiking, while other regions within the genome are recombined through gene flow $[1,3,4]$. Adaptive divergence in regions of the genome may be an important driving force in speciation, particularly when they create differences in

\footnotetext{
* Correspondence: btbarney@stanford.edu

${ }^{1}$ Department of Biology, Hopkins Marine Station, Stanford University, 120

Ocean View Boulevard, Pacific Grove, CA 92950, USA

Full list of author information is available at the end of the article
}

critical biological processes such as reproductive behavior and timing [1, 5], although the conditions under which these processes can occur may be limited $[6,7]$. A significant body of literature has been published on speciation with gene flow [8-11], and islands of divergence are often cited as a possible explanation of this phenomenon.

Within species, islands of divergence are associated with differential selection across variable environments. In the threespine stickleback within Lake Constance in Germany, for example, strong differentiation between migratory lake and resident stream ecotypes is associated with differentiation across a set of strongly linked genes [12]. Chromosomal inversions often lead to large 
regions of strong linkage disequilibrium [13], and studies of these inversion polymorphisms in various Drosophila species have shown that haplotype frequencies of these inversions change along environmental clines. For example, the In(3R)Payne inversion in D. melanogaster quickly evolved different frequencies across latitudes in Australia. Moreover, allele frequencies of the more equatorial haplotype of the inversion polymorphism have increased across the entire cline, suggesting a population adaptive response to increased temperature over 30 years [14-17]. A strikingly similar pattern occurs in D. subobscura, where the more southerly haplotype of the $\mathrm{O}$ inversion has increased in frequency over the last 15 to 30 years in all European populations tested [18]. In the yellow monkey flower (Mimulus guttatus), populations show differential fitness across coastal and inland habitats based on a set of genes linked within an inversion [19]. All in all, 'supergenes' are receiving more attention as a combination of genomic information and evolutionary theory shows how multi-locus selection and linkage can evolve to generate local adaptation [20-22].

The relationship between environmental conditions and genomic islands has also been studied in the Atlantic cod, Gadus morhua. Borza et al. (2010) and Hubert et al. (2010) developed a panel of 1500 SNPs derived from expressed sequence tags (EST) and used them to create linkage maps from pedigreed cod populations [23, 24]. They discovered three genomic islands of divergence that were highly associated with mean ocean temperatures across the northern Atlantic $[25,26]$. The same set of markers also describes ecotype divergence between migratory and stationary cod populations in Iceland and Norway [27], as well as small scale spatio-temporal patterns of population structure in Newfoundland [28, 29]. Using a custom SNP array from the recently published improved assembly of the cod genome [30], genomic differences were described between oceanic and coastal populations in the North Sea [31] and the Norwegian Sea [32]. In all these cases, genetic differences among populations at both oceanic and local scales are thought to be driven by environmental selection mediated by a suite of genes in several linkage groups, not by demographic barriers to gene flow. At smaller geographic and temporal scales, population genetic analyses of cod in the Gulf of Maine have focused on a suite of microsatellite and protein markers in a large number of individuals across many populations [33]. Most of the significant differences compiled in Zemeckis et al. [33] are from individual loci which appear to be under selection, rather than the result of neutral genetic structure between populations [34, 35].

To date, the loci within the cod linkage groups have been identified through breeding studies and classic quantitative trait loci (QTL) approaches. Moreover, the search for neutral genetic differentiation, outside the known linkage groups, has been based on relatively few loci compared to genome-level data sets. Both the searches for selected genes and those that might reflect neutral genetic differentiation have not yet taken advantage of full-genome approaches [36]. Here, we use high throughput sequencing and population genomic analyses to examine the degree and spatio-temporal distribution of linkage disequilibrium clusters throughout the genome of Atlantic cod in the Gulf of Maine and Georges Bank. We then use this information to eliminate linked genes from the analysis and use the remaining genes to test for patterns of neutral genetic differentiation.

Using complete genome sequencing, we describe a panel of 3,390,654 single nucleotide polymorphisms (SNPs) throughout the cod genome. Pairwise linkage analysis shows SNPs within 1031 genes falling into three cod 'supergenes', two of which show population divergence within the Gulf of Maine. Genes within these linkage blocks include DNA structural proteins and chromatin assembly genes, metabolic and catabolic genes, meiosis regulation and oocyte maturation genes, odorant receptors, egg coat structural proteins, heat shock proteins and many cell signaling genes that might be involved in environmental adaptation, habitat choice or mating. Once linkage disequilibrium blocks were identified and excluded from the analysis, our limited population data show no signs of neutral genetic differentiation in the three populations we sampled. Though our power to detect neutral structure is low with such few samples, comparison of many SNPs suggests that the signature of population differentiation is largely driven by shifts in the supergenes, possibly driven by present or past patterns of natural selection.

\section{Methods \\ Sample collection}

Fin-clip samples from adult Atlantic cod in spawning condition were collected and stored frozen prior to preparation of DNA libraries. A total of 31 individuals were sampled: 10 from Georges Bank, and 21 from the Gulf of Maine. Within the Gulf of Maine, the sample was subdivided into two groups, based on spawning season: 10 cod were sampled from a winter spawning group, and 11 cod from a spring spawning group (Fig. 1). These samples were taken from cod stocks in the northwestern part of Massachusetts Bay near Cape Ann, as studies of cod sampled from this region showed evidence of temporal population substructure based on breeding season [34, 35]. Samples were collected in March 2014 for the Georges Bank population, and in December 2013 and June 2014 for the Gulf of Maine winter and spring populations, respectively (Table 1). 


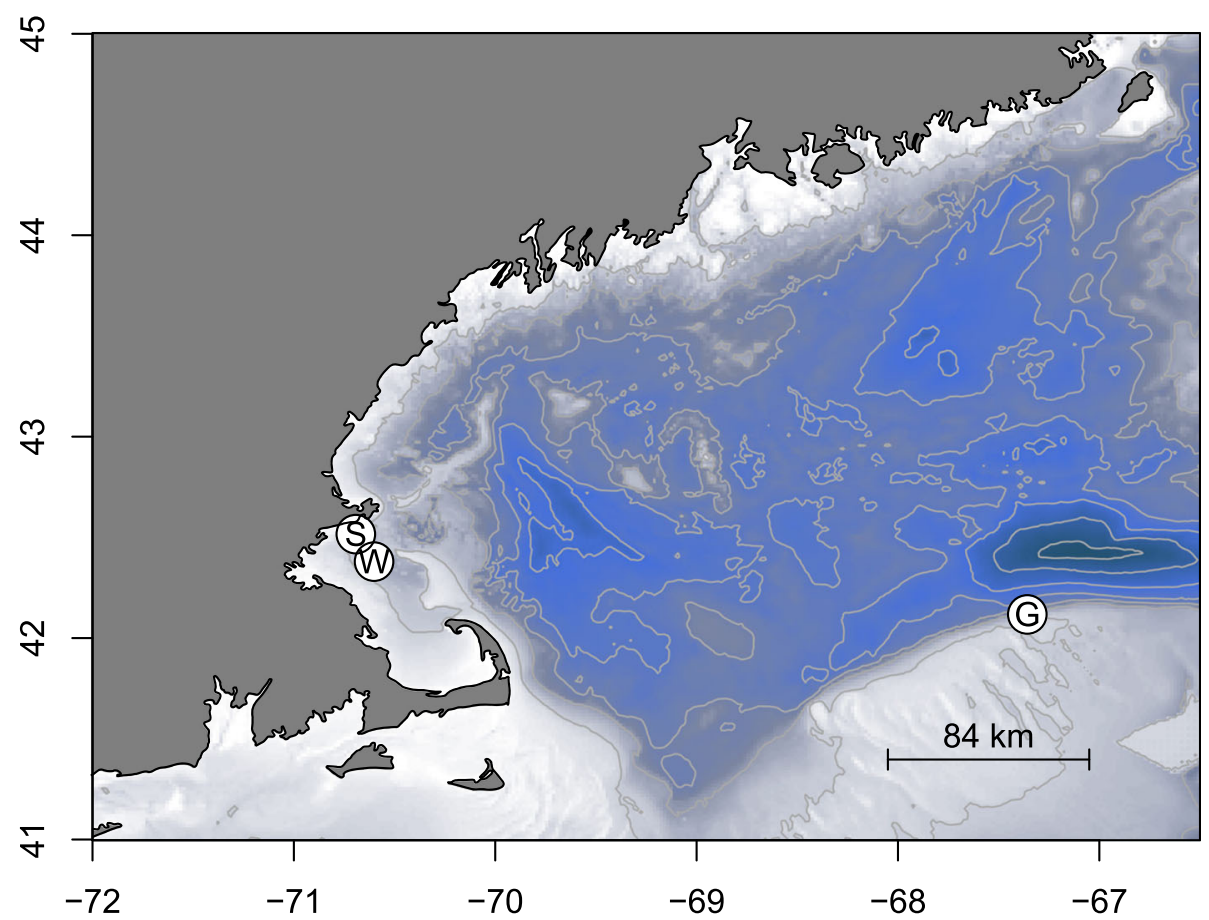

Fig. 1 Sampling locations used in this study. Twenty one adults in spawning condition were sampled in northwestern Massachusetts Bay within the Gulf of Maine. These were subdivided into 11 spring spawning cod (site S) and 10 winter spawning cod (site W). Ten adults in spawning condition were sampled from Georges Bank (site G). Figure was generated using the marmap package in $\mathrm{R}$

\section{DNA extraction, library preparation, sequencing}

Genomic DNA (gDNA) from each fin clip was prepared by digesting entire fin clips (approximately $1 \mathrm{~cm}^{2}$ ) in $500 \mu \mathrm{L}$ of Digestion Mixture $(1 \mu \mathrm{g} / \mu \mathrm{L}$ Proteinase $\mathrm{K}$, $50 \mathrm{mM}$ EDTA, 5\% Tween 20, 0.5\% Triton-X 100, $800 \mathrm{mM} \mathrm{GuHCl}, 0.5 \% \mathrm{SDS}$ ) at $50{ }^{\circ} \mathrm{C}$ for $1 \mathrm{~h}$. After digestion, the samples were centrifuged at 13,000 rpm for $10 \mathrm{~min}$; the supernatant was recovered, and then mixed with $50 \mu \mathrm{L}$ of $3 \mathrm{M}$ potassium chloride to precipitate the SDS. An equal volume of isopropyl alcohol was added to precipitate nucleic acids, and the pellet was washed with $70 \%$ ethanol. DNA/RNA was resuspended in $100 \mu \mathrm{L}$ of $10 \mathrm{mM}$ Tris- $\mathrm{HCl} \mathrm{pH} 8.0$ and treated with $5 \mu \mathrm{L}$ of $10 \mathrm{mg} / \mathrm{mL}$ RNase A. Another isopropyl alcohol precipitation was performed, then genomic DNA was isolated using AMPure XP beads. Total gDNA was resuspended and stored in TE buffer $(10 \mathrm{mM}$ Tris- $\mathrm{HCl}$, 1 mM EDTA, pH 8).

Table 1 Cod sampling summary

\begin{tabular}{lllll}
\hline Stock & Date & Samples (n) & Latitude & Longitude \\
\hline Gulf of Maine winter & Dec 2013 & 10 & 42.3813 & -70.6016 \\
Gulf of Maine spring & June 2014 & 11 & 42.5177 & -70.6921 \\
Georges Bank & Mar 2014 & 10 & 42.1192 & -67.3575 \\
\hline
\end{tabular}

Locations, dates, and number of samples collected at each sampling site
Sequencing libraries were prepared from $50 \mathrm{ng}$ of template DNA using the Illumina Nextera DNA library preparation kit (Illumina, San Diego, CA) following their standard protocol. Samples were individually barcoded to allow for multiplexing during sequencing. Each library was independently size selected using Ampure XP beads ( 0.6 volume beads to 1.0 volume DNA) and checked for adequate sizing via gel electrophoresis. Libraries were quantified by qPCR (KAPA Illumina Quantification Kit), then normalized and mixed in equimolar fashion.

One set of samples, consisting of five individuals from each sampled location, was sequenced on an Illumina HiSeq 2000 using 100 base paired end reads. Each batch of five samples from the original sample groupings were mixed and multiplexed across a distinct eight-lane HiSeq flowcell, resulting in approximately $80 x$ coverage across the genome (predicted size $\sim 800 \mathrm{Mb}$ ). The remaining samples (five winter, six spring, and five Georges Bank) were pooled and sequenced to less depth of coverage $(\sim 25 \mathrm{x})$ on three runs of an Illumina NextSeq 500 using 150 base paired end reads.

\section{Read mapping and variant calling}

Raw reads were trimmed of any residual adapter sequences, and low-quality base calls were removed using a baseline Phred score of 20 or less. Individual reads were retained if 
the length was $>70$ bases, and reads were re-paired using Trimmomatic [37]. Read pairs were then mapped to the reference genome file gadMor2.fasta [30] using bowtie2, with end-to-end alignment, 22 base seed length, two reseed attempts with no mismatches allowed in the seed, a seed interval of 17 bases, and all other settings at default values. Reads that became unpaired due to quality or trimming issues were mapped separately using the same parameters. The resulting mapped files were sorted and indexed using samtools [38] and duplicated reads were removed using Picard tools (http://broadinstitute.github.io/picard) for downstream SNP discovery and analysis.

SNP discovery was performed on individual samples using FreeBayes version 0.9.10-3-g47a713e, using the default settings [39]. Variants were filtered using vcftools to only include biallelic SNPs with a minimum minor allele frequency of 0.05 using vcftools version $0.1 .12 \mathrm{~b}$ [40]. Filtering by minor allele frequency ensures that SNP loci with extremely low diversity are removed from downstream analysis, as they are essentially uninformative. The resultant SNP list was further limited to those SNPs that were genotyped $(\mathrm{Q}>30)$ in all 31 samples.

\section{Data analysis}

We limited our analysis of linkage disequilibrium to linkage groups (hereafter LG) 2, 7, and 12, as these regions contain the three "islands of divergence" studied in this species. Within each LG, we extracted every 250th SNP, and performed pairwise linkage disequilibrium analysis using the LD function in the 'genetics' package (version 1.3.8.1) in $\mathrm{R}$ version 3.03 ( $\mathrm{R}$ development core team 2015). We determined the correlation coefficient (as $r$ ) between the paired genotypes of all 31 individuals, and reported the $r^{2}$ value to remove any arbitrary sign introduced by intermediate calculations. To examine the genomic extent of linkage disequilibrium effects within each LG, mean $r^{2}$ was calculated for every 250th SNP within each LG, as was pairwise $\mathrm{F}_{\mathrm{ST}}$. The edges of each linkage disequilibrium block (hereafter LD block) were located by finding the regions with high mean LD (mean $r^{2}>0.1$ ) and determining the first and last SNP with similar mean $r^{2}$ values to the block. As there are 250 SNPs between the first high LD SNP and its subset neighbor and untested linked loci are located within this space, we placed the LD block edges on the SNPs adjacent to the ones with the first and last high $\mathrm{LD}$ value.

Using the gene model information from the genome, we determined which genes were in each LD block, and performed enrichment tests of Gene Ontology (GO) categories of the genes within the resultant LD block using the weight01 algorithm within the 'topGO' package version 2.24.0 [41] in R. Additionally, all SNPs throughout the genome were binned into one of three classes; exon, intron, or intergenic. We determined the proportion of SNPs within each class inside each LD block and throughout the genome, to test if SNPs of any class were enriched within LD blocks.

To test for population differentiation, we filtered all SNPs for those found within exons and created subsets for analyses. For exonic SNPs on linkage groups 2, 7, and 12 we used all available exonic SNPs, each partitioned into two groups: those within the LD block and those outside of the block. For the remainder of the genome, we created a subset of every 10th exonic SNP. We focused solely on SNPs within exons for this analysis, as we are interested in both neutral population structure and adaptive divergence between spawning sites/times, and SNPs within the coding regions of genes can be used to examine both processes. From this subset of SNPs, Weir and Cockerham's pairwise $\mathrm{F}_{\mathrm{ST}}$ was calculated among the three populations using the wc function from the 'hierfstat' package $[42,43]$ within $R$. This was performed for exonic SNPs within each LD block, SNPs on the same linkage group but not in the LD block, and all exonic SNPs not on linkage groups 2, 7, or 12 . Principal component analysis (PCA) was performed on SNPs both within and outside of each LD block using the 'prcomp' function within the standard release package of $\mathrm{R}$.

To test for neutral population structure, we utilized the same subsets of exonic SNPs used for $\mathrm{F}_{\mathrm{ST}}$ and PCA analysis above. To avoid potential confounding selection with population structure, SNPs within the linkage blocks on LG02, LG07, and LG12 were removed from this dataset and tested separately. With these sets of SNPs, we tested for population structure using the admixture algorithm NGSadmix [44]. For each admixture analysis, we tested the model against a number of subpopulation divisions $(\mathrm{k}=2$ to $\mathrm{k}=5)$, and chose the $\mathrm{k}$ value that had the greatest likelihood among all $k$ values tested using the Evanno method [45].

\section{Results \\ Linkage disequilibrium block determination}

After mapping reads to the genome assembly, we identified 3,390,654 biallelic SNP variants with a minor allele frequency greater than 0.05 that were confidently genotyped in all 31 samples. There were 147,356 SNPs located on LG02, 204,124 SNPs on LG07, and 139,785 SNPs on LG12. To find the edges of the LD blocks, pairwise linkage disequilibrium (as $\mathrm{r}^{2}$ ) and $\mathrm{F}_{\mathrm{ST}}$ was calculated for every 250th SNP in LG02 (589 SNPs), LG07 (816 SNPs), and LG12 (559 SNPs). From these data, mean pairwise $r^{2}$ values were determined for each SNP (Fig. 2). Each LD block within a linkage group shows a highly contiguous and sharply defined region of elevated mean linkage disequilibrium (mean $r^{2}>0.1$ ) in comparison with the surrounding SNPs (mean $r^{2} \sim 0.03$ to 0.04). $\mathrm{F}_{\mathrm{ST}}$ was less useful in determining the edges of $\mathrm{LD}$ 


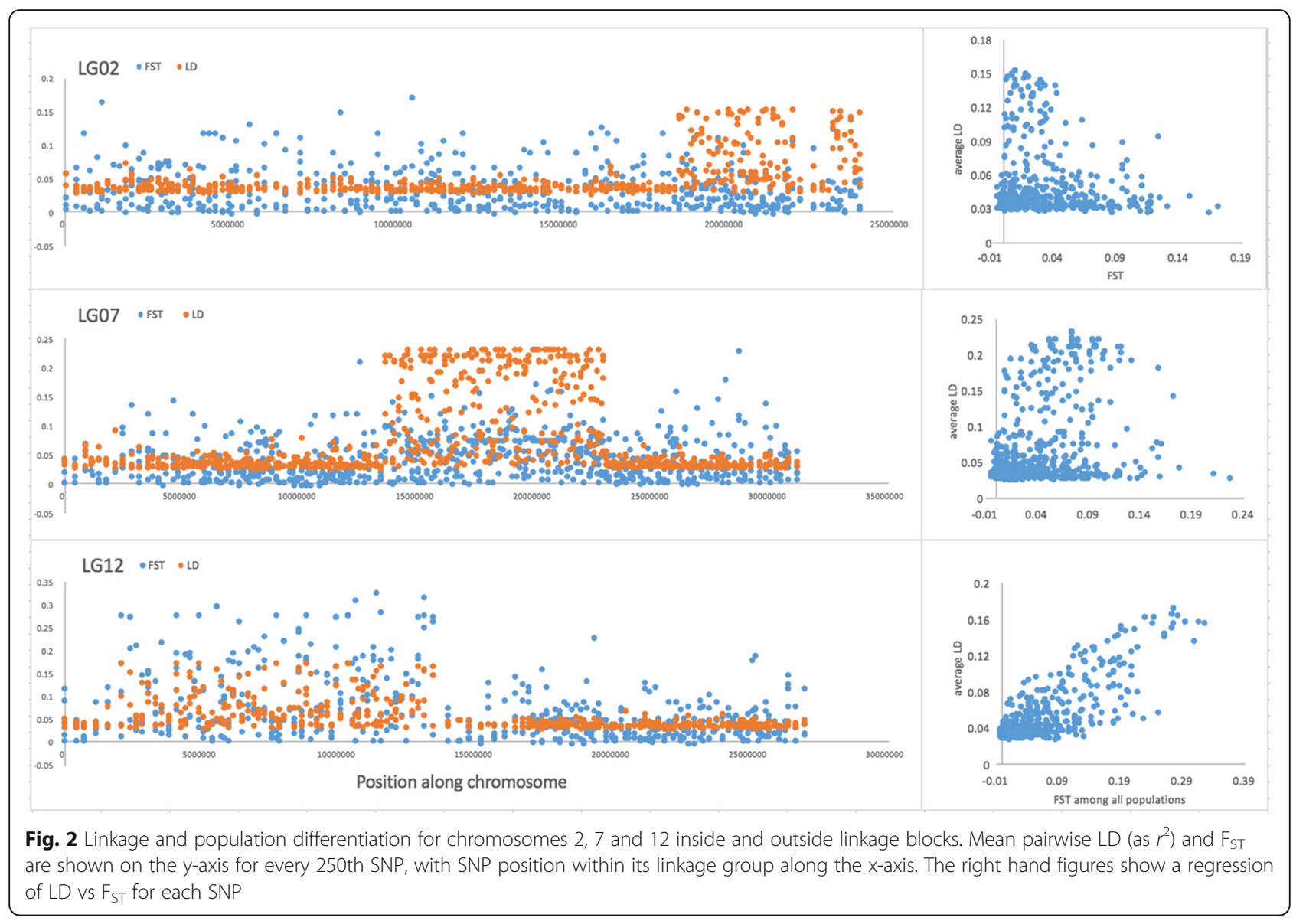

blocks, as this varied over wide ranges on SNPs within linkage groups 2 and 7. Interestingly, $\mathrm{F}_{\mathrm{ST}}$ and LD appear correlated in linkage group 12 (Fig. 2, bottom right), perhaps due both to the large extent of the linkage region and the high degree of differentiation seen in this sample set between the winter spawning cod and Georges Bank populations in the linked loci.

To capture potentially linked loci between the subset SNP loci, the starting base for each LD block was identified as the SNP immediately before the first SNP with a mean $r^{2}>0.1$ in the LD block, and the ending base was the first SNP after the last base with a mean $r^{2}>0.1$ in the LD block, resulting in LD blocks of $5.6 \mathrm{Mb}$ (LG02), 9.3 $\mathrm{Mb}$ (LG07) and 11.6 Mb(LG12) in length (Table 2). There was a linkage gap of about 2 million bases in LG02, inside which SNPs were not linked to one another

Table 2 Linkage disequilibrium block extent, and approximate length of each block

\begin{tabular}{lllll}
\hline & Starting base & Ending base & \# genes & length \\
\hline LG02 & $18,442,392$ & $24,053,701$ & 294 & $5.6 \mathrm{Mb}$ \\
LG07 & $13,743,341$ & $23,039,143$ & 306 & $9.3 \mathrm{Mb}$ \\
LG21 & $2,039,256$ & $13,611,225$ & 437 & $11.6 \mathrm{Mb}$ \\
\hline
\end{tabular}

or to the SNPs in the rest of the linkage group (Fig. 2, top left). For LG02, 58 of the 174 SNPs within the LD block $(\sim 33 \%)$ were strongly linked (mean pairwise $r^{2}>0.1$ ), while 170 of the 312 SNPs ( 54\%) in LG07 and 42 of the 246 SNPs ( 17\%) in LG12 were strongly linked. Our findings agree with regions of linkage disequilibrium identified previously by EST-derived SNPs [23, 25-27] and Illumina SNP arrays [31, 32]. However, our analysis provides a higher degree of resolution of both the range and edges of these LD blocks, as previous work has described LD blocks in terms of relative centimorgans(cM) rather than actual base positions.

\section{Genetic content of LD blocks}

Using the available gene models from the cod genome and the start and stop base positions for each LD block described above, we determined which genes were within each LD block. Of the 1095 genes on LG02, 294 were found within the boundaries of the LD block. GO enrichment analysis on genes within this LD block showed several overlapping GO terms with significant enrichment ( $p$ adj $<0.01$ after false-discovery rate correction) centered on genes involved with DNA/chromatin structuring (Table 3). Genes within the significant GO terms consisted 
Table 3 GO enrichment analysis of linkage disequilibrium block on LG02

\begin{tabular}{|c|c|c|c|c|c|}
\hline GO.ID & Term & Annotated & Significant & Fisher pval & padj \\
\hline GO:0006325 & chromatin organization & 142 & 18 & $1.70 \mathrm{E}-14$ & $2.52 \mathrm{E}-11$ \\
\hline GO:0065004 & protein-DNA complex assembly & 105 & 16 & $2.80 \mathrm{E}-14$ & $2.52 \mathrm{E}-11$ \\
\hline GO:0071824 & protein-DNA complex subunit organization & 105 & 16 & $2.80 \mathrm{E}-14$ & $2.52 \mathrm{E}-11$ \\
\hline GO:0051276 & chromosome organization & 178 & 19 & 7.10E-14 & $4.76 \mathrm{E}-11$ \\
\hline GO:0006334 & nucleosome assembly & 100 & 15 & $2.50 \mathrm{E}-13$ & $9.39 \mathrm{E}-11$ \\
\hline GO:0031497 & chromatin assembly & 100 & 15 & $2.50 \mathrm{E}-13$ & $9.39 \mathrm{E}-11$ \\
\hline GO:0034728 & nucleosome organization & 100 & 15 & $2.50 \mathrm{E}-13$ & $9.39 \mathrm{E}-11$ \\
\hline GO:0006333 & chromatin assembly or disassembly & 101 & 15 & $2.90 \mathrm{E}-13$ & 9.57E-11 \\
\hline GO:0006323 & DNA packaging & 106 & 15 & $6.00 \mathrm{E}-13$ & 1.77E-10 \\
\hline GO:0071103 & DNA conformation change & 112 & 15 & $1.40 \mathrm{E}-12$ & $3.65 \mathrm{E}-10$ \\
\hline GO:0034622 & cellular macromolecular complex assembly & 180 & 16 & $1.30 \mathrm{E}-10$ & $3.26 \mathrm{E}-08$ \\
\hline GO:0043933 & macromolecular complex subunit organization & 324 & 20 & 3.90E-10 & 8.64E-08 \\
\hline GO:0006461 & protein complex assembly & 244 & 17 & $1.50 \mathrm{E}-09$ & 2.91E-07 \\
\hline GO:0070271 & protein complex biogenesis & 244 & 17 & $1.50 \mathrm{E}-09$ & 2.91E-07 \\
\hline GO:0065003 & macromolecular complex assembly & 258 & 17 & 3.60E-09 & $6.38 \mathrm{E}-07$ \\
\hline GO:0071822 & protein complex subunit organization & 271 & 17 & 7.50E-09 & $1.24 \mathrm{E}-06$ \\
\hline GO:0022607 & cellular component assembly & 308 & 18 & 7.90E-09 & $1.24 \mathrm{E}-06$ \\
\hline GO:0006996 & organelle organization & 432 & 21 & $1.00 \mathrm{E}-08$ & $1.50 \mathrm{E}-06$ \\
\hline GO:0044085 & cellular component biogenesis & 339 & 18 & $3.50 \mathrm{E}-08$ & 4.95E-06 \\
\hline GO:0016043 & cellular component organization & 636 & 22 & $1.70 \mathrm{E}-06$ & 0.00023 \\
\hline GO:0071840 & cellular component organization or biogenesis & 666 & 22 & $3.70 \mathrm{E}-06$ & 0.00047 \\
\hline GO:0042157 & lipoprotein metabolic process & 32 & 5 & $2.70 \mathrm{E}-05$ & 0.0032 \\
\hline GO:0044711 & single-organism biosynthetic-process & 295 & 9 & 0.0061 & 0.7060 \\
\hline GO:0006897 & endocytosis & 35 & 3 & 0.0071 & 0.7895 \\
\hline GO:0043543 & protein acylation & 12 & 2 & 0.0078 & 0.8318 \\
\hline GO:0016568 & chromatin modification & 38 & 3 & 0.0089 & 0.8645 \\
\hline
\end{tabular}

Using topGO, (in R), enrichment analysis shows multiple GO categories that may be enriched in the LD block from LG02, mostly centered on DNA/chromatin structuring. Total number of annotated genes in each GO category is shown, as well as those within the LD block that are significant (Fisher test $p<0.01$ ). Multiple-test correction (calculated as false-discovery rate, ' $\mathrm{fdr}$ ') is shown in $p$-adj column, $p$-values $<0.05$ shown in bold

primarily of histone proteins involved in the assembly of the nucleosome: 14 of the 27 genes in the significant GO terms coded for histone proteins (Table 4). Additionally, two histone modification genes, histone-lysine $\mathrm{N}$-methyltransferase and histone deacetylase are also located within this linkage block.

Within the 1166 genes on LG07, 306 were located within its LD block, including genes involved with signaling and metabolic processes, though no GO categories remained significant after false-discovery rate correction (Table 5). Similarly, the LD block within LG12 contained 437 genes out of the 975 genes in that linkage group, and no significant enrichment of any GO categories remained after false-discovery rate correction (Table 6). Specific genic content within each block can be found in the Additional files 1, 2, 3 for this publication.

In addition to the genic content within each LD block, we evaluated the distributions of SNP locations (classified as intergenic, exon, or intron) of all SNPs located on linkage groups 1 through $23(n=3,283,653$ SNPs). This number is slightly smaller than the total number of SNPs passing our filters $(n=3,390,654)$ due to unused SNPs located on the few remaining unscaffolded contigs as well as mitochondrial DNA, yet still represents $97 \%$ of all identified SNPs. Proportions of each SNP type were calculated within each LD block, as well as the remaining SNPs not on linkage groups 2,7 , or 12 (Table 6). SNP density (as \# SNPs/base) was also determined within each LD block and in the remainder of the genome as above. For the LD block on LG02, there was both an increase in overall SNP density (from 5.66e-03 to 7.72e-03, a 36\% increase) and an increase in the proportion of exonic SNPs (from 0.065 to 0.089 , a $37 \%$ increase) compared to genome-wide values. We also found a $50 \%$ increase over the genome-wide SNP density within the LD block on LG07 (from 5.66e-03 to 8.36e-03 SNPs/base), though there were no significant 
Table 4 Genes in significant GO terms within LD block on LG02

\begin{tabular}{|c|c|c|}
\hline Gene & Starting base & Gene description \\
\hline GAMO_00059458 & 18475327 & zgc:112234: Histone H2B 1/2 (Danio rerio) \\
\hline GAMO_00059459 & 18476591 & Histone H2A (Oncorhynchus mykiss) \\
\hline GAMO_00059461 & 18489582 & TGas006m08.1: Histone H4 (Xenopus tropicalis) \\
\hline GAMO_00059462 & 18491420 & Histone H2A (Oncorhynchus mykiss) \\
\hline GAMO_00059465 & 18506398 & Histone H2A (Oncorhynchus mykiss) \\
\hline GAMO_00059556 & 19188916 & STRA13: Centromere protein X (Homo sapiens) \\
\hline GAMO_00059593 & 19396062 & Crebbp: CREB-binding protein (Rattus norvegicus) \\
\hline GAMO_00059696 & 20010135 & NMT1: Glycylpeptide N-tetradecanoyltransferase 1 (Homo sapiens) \\
\hline GAMO_00059920 & 21260662 & Coro1b: Coronin-1B (Mus musculus) \\
\hline GAMO_00059924 & 21287842 & suv420h2: Histone-lysine N-methyltransferase SUV420H2 (Xenopus laevis) \\
\hline GAMO_00059998 & 21707418 & KCNA1: Potassium voltage-gated channel subfamily A member 1 (Homo sapiens) \\
\hline GAMO_00060305 & 23130440 & tmem11: Transmembrane protein 11\%2C mitochondrial (Danio rerio) \\
\hline GAMO_00060314 & 23170545 & Histone H2A (Oncorhynchus mykiss) \\
\hline GAMO_00060318 & 23177224 & Histone H2A (Oncorhynchus mykiss) \\
\hline GAMO_00060319 & 23198635 & Histone H3 (Urechis caupo) \\
\hline GAMO_00060320 & 23199855 & Histone H2A (Oncorhynchus mykiss) \\
\hline GAMO_00060321 & 23201514 & TGas006m08.1: Histone H4 (Xenopus tropicalis) \\
\hline GAMO_00060322 & 23202645 & Histone H3 (Urechis caupo) \\
\hline GAMO_00060324 & 23204017 & Histone H2B (Salmo trutta) \\
\hline GAMO_00060364 & 23359510 & Pigq: Phosphatidylinositol N-acetylglucosaminyltransferase subunit Q (Mus musculus) \\
\hline GAMO_00060373 & 23393752 & Histone H2A (Oncorhynchus mykiss) \\
\hline GAMO_00060375 & 23400842 & TGas006m08.1: Histone H4 (Xenopus tropicalis) \\
\hline GAMO_00060376 & 23401993 & Histone H3 (Urechis caupo) \\
\hline GAMO_00060390 & 23480761 & HDAC9: Histone deacetylase 9 (Homo sapiens) \\
\hline GAMO_00060462 & 23855114 & APOL3: Apolipoprotein L3 (Homo sapiens) \\
\hline GAMO_00060465 & 23866729 & APOL3: Apolipoprotein L3 (Homo sapiens) \\
\hline GAMO_00060467 & 23891523 & APOL3: Apolipoprotein L3 (Homo sapiens) \\
\hline
\end{tabular}

While several GO terms were significantly enriched, many of them collapsed to the same set of DNA structural genes, including several genes encoding all four major histone proteins that make up the nucleosome

Table 5 GO enrichment analysis of linkage disequilibrium block on LG07

\begin{tabular}{|c|c|c|c|c|c|}
\hline GO.ID & Term & Annotated & Significant & Fisher pval & $p$-adj (fdr) \\
\hline GO:0006855 & drug transmembrane transport & 5 & 2 & 0.0014 & 1 \\
\hline GO:0006066 & alcohol metabolic process & 24 & 3 & 0.0028 & 1 \\
\hline GO:0015893 & drug transport & 8 & 2 & 0.0038 & 1 \\
\hline GO:0042493 & response to drug & 8 & 2 & 0.0038 & 1 \\
\hline GO:0044765 & single-organism transport & 1098 & 23 & 0.0044 & 1 \\
\hline GO:0023051 & regulation of signalling & 275 & 9 & 0.0054 & 1 \\
\hline GO:1902578 & single-organism localization & 1118 & 23 & 0.0055 & 1 \\
\hline GO:1901615 & $\begin{array}{l}\text { organic hydroxy compound } \\
\text { metabolic process }\end{array}$ & 31 & 3 & 0.0058 & 1 \\
\hline GO:0006071 & glycerol metabolic process & 10 & 2 & 0.0059 & 1 \\
\hline GO:0019400 & alditol metabolic process & 10 & 2 & 0.0059 & 1 \\
\hline
\end{tabular}

Using topGO, (in R), enrichment analysis shows multiple GO categories that may be enriched in the LD block from LG07, including genes involved with signal transport and metabolic processes. Total number of annotated genes in each GO category is shown, as well as those within the LD block that are significant (Fisher test $p<0.01$ ). Multiple-test correction (calculated as false-discovery rate, ' $\mathrm{fdr}$ ') is shown in $p$-adj column 
Table 6 GO enrichment analysis of linkage disequilibrium block on LG12

\begin{tabular}{|c|c|c|c|c|c|}
\hline GO.ID & Term & Annotated & Significant & Fisher pval & $p$-adj (fdr) \\
\hline GO:0051648 & vesicle localization & 4 & 2 & 0.002 & 1 \\
\hline GO:0051650 & establishment of vesicle localization & 4 & 2 & 0.002 & 1 \\
\hline GO:0009057 & macromolecule catabolic process & 144 & 8 & 0.0051 & 1 \\
\hline GO:0051656 & establishment of organelle localization & 7 & 2 & 0.0067 & 1 \\
\hline GO:0051640 & organelle localization & 8 & 2 & 0.0088 & 1 \\
\hline
\end{tabular}

Using topGO, (in R), enrichment analysis shows multiple GO categories that may be enriched in the LD block from LG12. Total number of annotated genes in each GO category is shown, as well as those within the LD block that are significant (Fisher test $p<0.01$ ). Multiple-test correction (calculated as false-discovery rate ' $\mathrm{fdr}$ ') is shown in $p$-adj column

changes in the proportions of SNP type within this group and no deviations from expected polymorphism rates in LG12 (Table 7).

\section{Differentiation among sampled populations}

The subsets of exonic SNPs used for population structure analyses contained a total of 54,030 SNPs. $F_{\mathrm{ST}}$ analysis of linkage group 2 used 11,241 SNPs (3874 in LD block, 7367 outside), linkage group 7 used 13,839 SNPs (4920/8919) and linkage group 12 used 8835 SNPs (4343/4492). The remainder of the genome used a subset of exonic SNPs $(n=20,115$ SNPs). Mean pairwise $\mathrm{F}_{\mathrm{ST}}$ for all loci tested $(n=54,030)$ ranged between 0.0062 (spring spawners vs. Georges Bank) and 0.0297 (winter spawners vs. Georges Bank). Mean pairwise $\mathrm{F}_{\mathrm{ST}}$ analysis of each SNP subset tested shows a trend of increased $\mathrm{F}_{\mathrm{ST}}$ values within some LD blocks as compared to the exonic SNPs on the same linkage group. Mean pairwise $\mathrm{F}_{\mathrm{ST}}$ for each comparison is shown in Table 8.

We also examined patterns of $\mathrm{F}_{\mathrm{ST}}$ and $\mathrm{LD}$ along each of the chromosomes with linkage blocks. $\mathrm{F}_{\mathrm{ST}}$ among all three populations was highly correlated with linkage disequilibrium in LG02 and LG12 (Fig. 2, right upper and lower), but was only moderately correlated in LG07 (Fig. 2, right middle). Linkage was highest in LG07, whereas $\mathrm{F}_{\mathrm{ST}}$ was higher within the linkage groups LG07 and LG12. LG07 also shows a number of chromosomal areas outside the main region of linkage that have occasional high $\mathrm{F}_{\mathrm{ST}}$, and a low relationship overall between linkage and $\mathrm{F}_{\mathrm{ST}}$ (Fig. 2, left middle). Variation in the relationship between linkage and $\mathrm{F}_{\mathrm{ST}}$ suggests other patterns of selection acting on genes on LG07 that would warrant future research.

The LD block on linkage group 2 showed a significant increase in higher FST values when comparing spring spawning cod to the winter spawning cod (Fig. 3, A1, mean $\mathrm{F}_{\mathrm{ST}}=0.1166$ ) as well as between winter spawners and Georges Bank cod (Fig. 3, A3, mean $\mathrm{F}_{\mathrm{ST}}=0.0981$ ) using a Kolmogorov-Smirnov two-sided test on the $\mathrm{F}_{\mathrm{ST}}$ distributions $(p<1 \mathrm{e}-10$, both comparisons). The LD block on linkage group 7 was also genetically divergent between winter spawners and Georges Bank cod (Fig. 3, $\mathrm{B} 3$, mean $\mathrm{F}_{\mathrm{ST}}=0.0895$, KS test $p<1 \mathrm{e}-10$ ). However, the highest differentiation was found between winter spawners and Georges Bank cod within the LD block on LG12 (mean $\mathrm{F}_{\mathrm{ST}}=0.1726$, KS test $p<1 \mathrm{e}-10$ ), with a broader, longer-tailed distribution of $\mathrm{F}_{\mathrm{ST}}$ values. Mean pairwise $F_{S T}$ values for exonic SNPs on the same linkage groups but not within LD blocks ranged between 0.0001 and 0.0036 , and mean $F_{\mathrm{ST}}$ values for the remainder of the genome ranged between 0 and 0.0003 , which is consistent with one large, outbreeding population.

Principal component analysis was used on SNPs within each LD block, and on SNPs on the same linkage group, but outside the LD block (Fig. 4). In all cases, PCA on SNPs within LD blocks showed three distinct clusters along principal component axis 1, correlating with the three possible genotypes of that LD block (Fig. 4, left). This is in contrast to the remaining exonic SNPs on the same linkage group (Fig. 4, right), where no real clustering is seen between sampled populations, suggesting that all significant divergence is centralized on the three main blocks of linkage disequilibrium.

Table 7 Comparison of genome contents and population divergence of cod chromosome linkage groups 2, 7 and 12

\begin{tabular}{lccccccccccc}
\hline Group & \# SNPS & $\begin{array}{l}\text { Block } \\
\text { length } \\
\text { (Mb) }\end{array}$ & SNP linked \# genes & $\begin{array}{l}\text { Genes } \\
\text { in LD }\end{array}$ & $\begin{array}{l}\text { SNP } \\
\text { density }\end{array}$ & $\begin{array}{l}\text { proportion } \\
\text { exonic SNPs }\end{array}$ & $\begin{array}{l}\text { Fst in LD FST vs LD } \\
\text { block }\end{array}$ & $\begin{array}{l}\text { FST outside } \\
\text { LD block }\end{array}$ & Notes \\
\hline LG02 & 43,341 & 5.6 & 33 & 1095 & 294 & $7.72 \mathrm{E}-03$ & 0.089 & 0.098 & negative & 0.0025 & discontinuous block \\
LG07 & 77,751 & 9.3 & 54 & 1166 & 306 & $8.36 E-03$ & 0.064 & 0.089 & positive & 0.0004 & marked lack of SNPs \\
LG12 & 61,251 & 11.6 & 17 & 975 & 437 & $5.29 E-03$ & 0.071 & 0.173 & higly positive 0.0022 & $\begin{array}{c}\text { adjacent to linkage } \\
\text { block }\end{array}$
\end{tabular}


Table 8 Mean pairwise FST comparisons between sampled populations

\begin{tabular}{llll}
\hline & Spring vs. Winter & Spring vs. GB & Winter vs. GB \\
\hline LG02 in LD & $\mathbf{0 . 1 1 6 6}$ & -0.0189 & $\mathbf{0 . 0 9 8 1}$ \\
LG02 out & 0.0025 & 0.0021 & 0.0016 \\
LG07 in LD & -0.0062 & 0.0121 & $\mathbf{0 . 0 8 9 5}$ \\
LG07 out & 0.0004 & $-7.43 E-05$ & 0.0001 \\
LG12 in LD & 0.0231 & 0.0766 & $\mathbf{0 . 1 7 2 6}$ \\
LG12 out & 0.0022 & 0.0021 & 0.0036 \\
Genome & 0.0001 & -0.0003 & 0.0003
\end{tabular}

Mean pairwise $\mathrm{F}_{S T}$ calculations for each sampled population show some patterns of divergence between sampled populations, with high mean $\mathrm{F}_{\mathrm{ST}}$ values between winter spawners and Georges Bank cod in all three LD blocks (shown in bold). LG02 is also highly divergent between spring and winter spawners

Finally, we found no evidence for neutral population structure when using the population admixture analysis algorithm NGSadmix. When using $\mathrm{k}=2$ for SNPs within each linkage disequilibrium block, we found that individual admixture predictions perfectly matched the known genotypes of that individual in the linked loci with the LD block, with homozygous individuals binned into the two populations and heterozygotes having 50/50 admixture, as expected. However, all runs for the entire exonic SNP dataset predicted $\mathrm{k}=1$ as the most likely population model using the Evanno method of comparing model likelihood scores [45]. All models of $\mathrm{k}=2$ or higher predicted a high proportion of admixture for all samples and showed no clear differentiation between sampled locations.

\section{Discussion}

In this study, we used whole genome resequencing and single nucleotide polymorphism discovery to describe patterns of genome-wide linkage disequilibrium in the Atlantic cod (Gadus morhua) at small spatio-temporal scales within the Gulf of Maine and Georges Bank. Through sequencing and linkage disequilibrium analysis, we found three large clusters of linkage disequilibrium spanning multiple genes, in agreement with the known
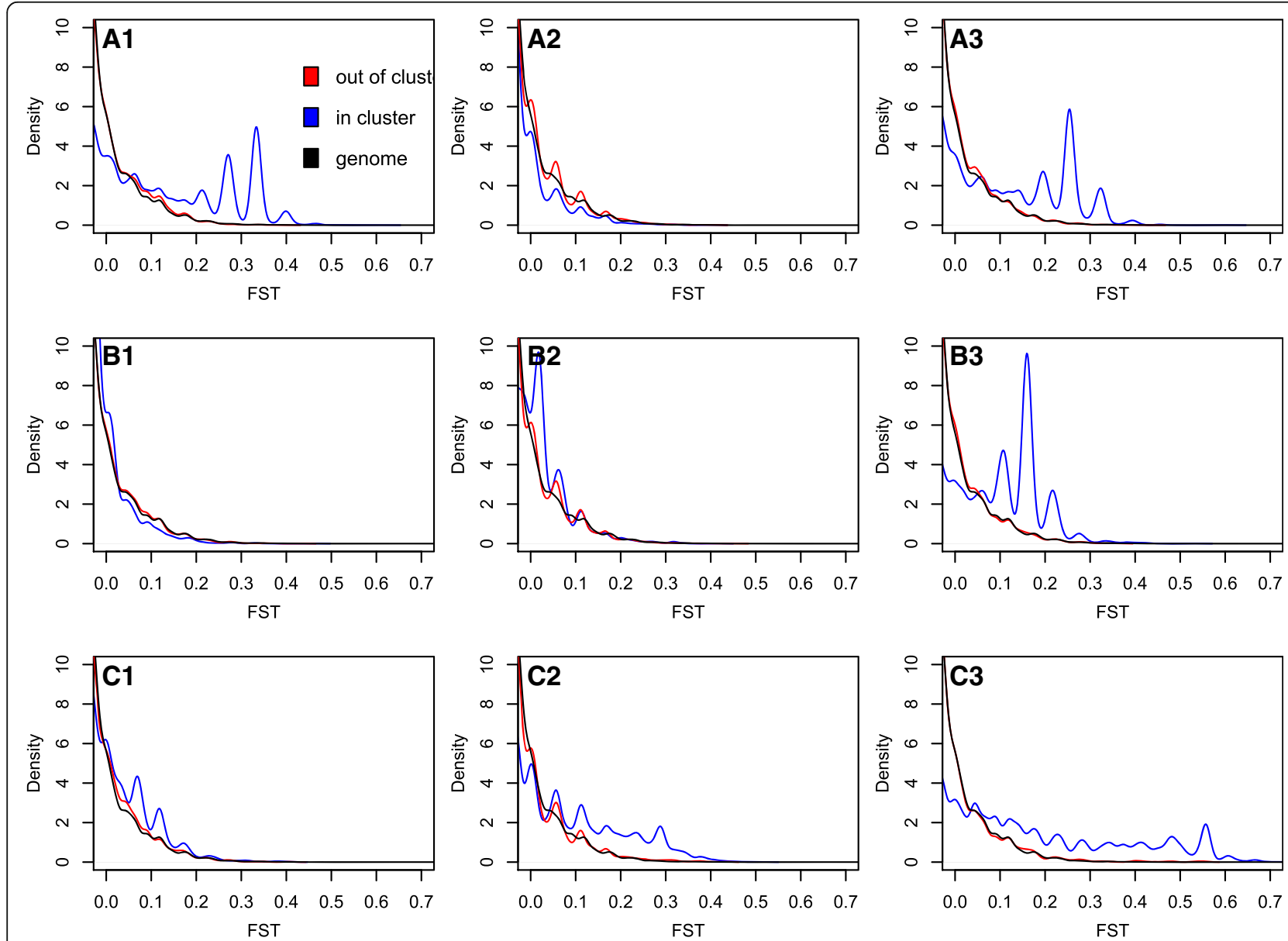

Fig. 3 Distributions of pairwise $F_{S T}$ within LD blocks between sampled populations. Pairwise $F_{S T}$ was determined within LD blocks (blue), on the same LG but not in the LD block (red), as well as the remainder of the genome (black) for LD blocks on LG02 (top row) LG07 (middle row), and LG12 (bottom row). Column 1 is the pairwise FST comparison between spring and winter spawners, column 2 is spring vs. Georges Bank, and column 3 is winter vs. Georges Bank 

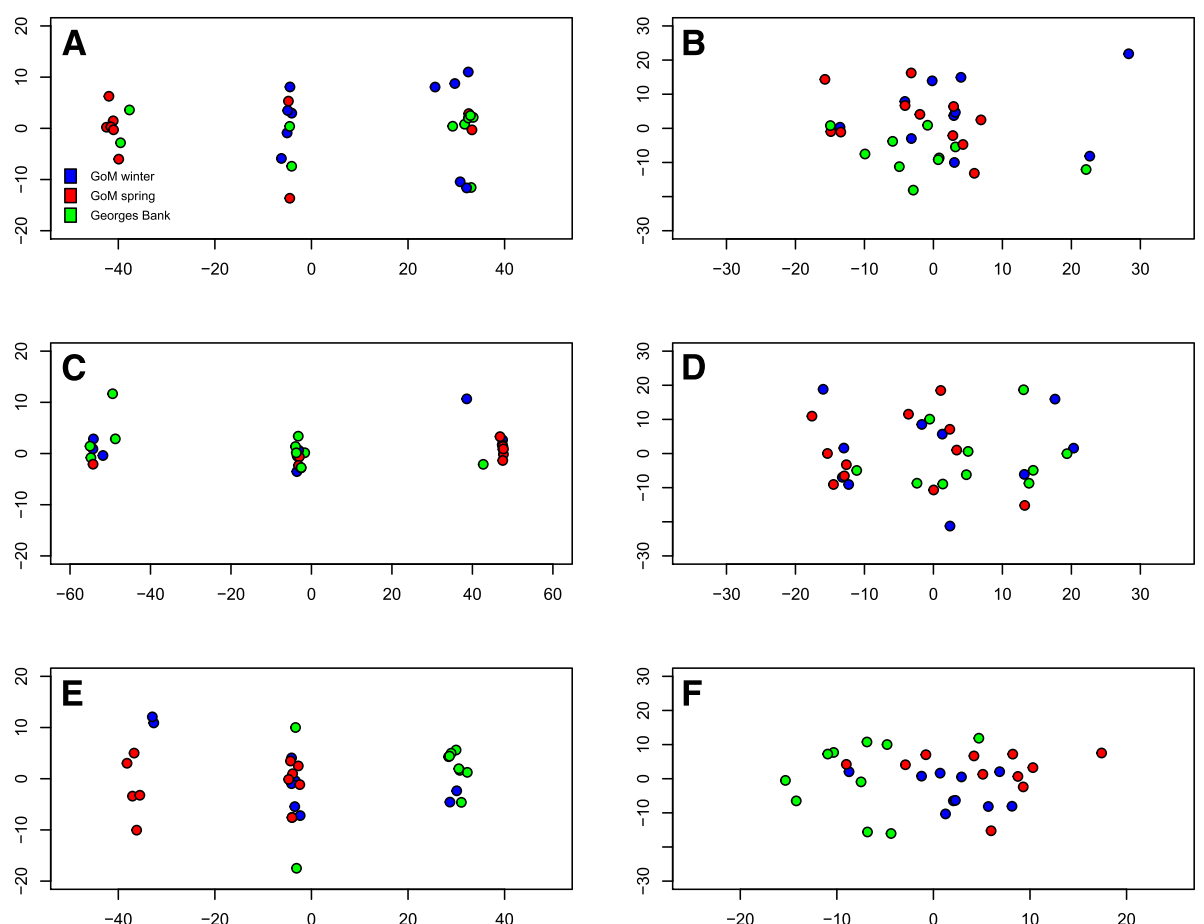

Fig. 4 Principal component analyses. PCA was performed on exonic SNPs from LG02 (top row), LG07 (middle row), and LG12 (bottom row). SNPs within the LD block on that LG are shown on the left, SNPs outside the LD block on the right. In all cases, as expected, SNPs within each LD block separate into three distinct vertical clusters along PC axis 1, indicating the three genotypes that individuals might possess for each LD block. This separation is lost, however, for PCA on SNPs outside the LD block, but in the same linkage group. Note that SNPs outside of the LD block on LG07 (D, above) may signify the presence of another, undiscovered linkage disequilibrium cluster, as the pattern appears diffusely similar to that in C

relative positions of these LD clusters from previously published studies of genomic "islands of divergence" in cod [23, 25-27, 31, 32]. Earlier work had identified such clusters through a set of pedigree-based linkage maps of EST-derived SNPs and microsatellites in controlled crosses. Using custom microarrays, gene lists have been developed to describe the genic content of these linkage regions [32]. Specifically, the LD blocks on LG02 and LG07 have been shown to contain 189 and 297 genes, respectively. Our analysis augments these gene lists to contain 294 and 306 genes, includes an additional 437 genes on LG12, and shows that the extent and genic content of linkage disequilibrium blocks can be detected de novo by deep sequencing of wild-caught individuals.

The Atlantic cod genomic islands of divergence are associated with strong natural selection across the Atlantic [25, 26], in the Atlantic Canadian region [27-29], and in the Norwegian Sea $[31,32,46]$. In our samples, large blocks of linkage disequilibrium within LG02, LG07, and LG12 showed a differential pattern of genetic differentiation, with the LD block in LG02 separating between spring and winter spawners within the Gulf of Maine, and the LD blocks within all three linkage groups separating between the winter spawners and Georges Bank cod. This is in direct contrast to SNPs on the same linkage group but outside the LD block, suggesting that the observed differentiation within LD blocks may be due to selection acting on the LD blocks as a unit.

\section{Temperature and linkage clusters}

Local adaptation is not uncommon in marine systems [47]. Studies typically examine local adaptation across large geographic scales, often working at ranges of thousands of kilometers [48-51]. However, evidence is accumulating that highly local adaptation in high gene flow species may be more common than previously thought in both terrestrial and marine systems [52, 53]. Indeed, within Atlantic cod, the genomic region in linkage map LG02 (at $\sim 50$ centimorgans) has been shown to have a clinal relationship to average water temperature across the Atlantic, with cod from both Georges Bank and Galway Bay, Ireland ("warmer" locations, mean bottom temp. $\sim 8{ }^{\circ} \mathrm{C}$ ) largely having one linkage haplotype, and cod from both Newfoundland and Norway ("colder" locations, mean bottom temp. $\sim 0{ }^{\circ} \mathrm{C}$ ) having the alternate haplotype [26]. We found the same LD block diverging between spring and winter spawning cod within the Gulf of Maine, where near-surface temperatures can vary from $3{ }^{\circ} \mathrm{C}$ in late winter to $10{ }^{\circ} \mathrm{C}$ in early autumn [54]. Such seasonal variation may facilitate the temporal 
partitioning of subpopulations of cod if they are adapted to spawning at certain temperature ranges [55].

\section{Nature of selection in putative 'supergenes'}

Gene located within the LD block of linkage group 2 showed a significant enrichment for genes associated with DNA and chromatin structuring.

This cluster was most divergent between the spring and winter spawning cod within the Gulf of Maine, but was also divergent between winter spawners and Georges Bank. Historically, this linkage disequilibrium block has been shown to differentiate between stationary and migratory cod populations in Norway [31,32], and have strong allelic associations with seawater temperatures across the Atlantic [26].

It is well understood that temperature plays a large role in the stress response of organisms, including the maintenance of DNA stability [56]. Chromatin plays an important role in the structuring and stability of the DNA molecule, controlling the regulation of gene expression either by relaxing and exposing regions of DNA for easier transcription or by tightening and shutting down transcription. Recently, expression changes in genes involved in chromatin organization and the regulation of DNA replication have been shown to be one of the earliest larval responses to temperature stress in larval zebrafish [57]. In our GO enrichment analysis for LG02, we found several of the major histone proteins $(\mathrm{H} 2 \mathrm{~A} / \mathrm{B}, \mathrm{H} 3 \mathrm{a}$, and $\mathrm{H} 4 \mathrm{~A}$ ), as well as a histone methyltransferase, which methylates the chromatin to alter the ability to transcribe genes, and a histone deacetylase, which removes acetyl groups from chromatin which dampens gene expression. This clustering of histone structural proteins and associated histone methylation and deacetylation genes within the linkage block on LG02 fits the concept of a 'supergene,' having several linked loci, inherited as a single unit, with enrichment for related functional genes as a 'complex phenotype.'

In addition to functional enrichment for histonerelated genes, SNPs within the LD block on LG02 are also more likely to be found within exons, as compared to the rest of the genome. $8.9 \%$ of the 43,441 SNPs in this linkage block are found in exons, which is a $37 \%$ increase over the $6.5 \%$ of $2,792,388$ SNPs in the rest of the genome. The increase in exonic SNPs, together with a $36 \%$ increase in overall SNP density within this LD block, suggests an accumulation and maintenance of genetic variation within this LD block. This is consistent with a balanced, older inversion polymorphism, as time has allowed mutation to accumulate, and selection along with protection from recombination maintains the entire region as a unit, protecting minor deleterious mutations from removal.
While no significant enrichment for GO terms was found in the LD blocks within LG07 or LG12, we know that these genomic regions have been shown to diverge strongly between coastal stationary and offshore migratory cod in Norway [31, 32]. The selection acting on these regions may be acting on one or a few genes of high importance within each LD block, and individuals inherit the region in its entirety due to the repressed recombination of inversion polymorphisms. In our study, we find differentiation between winter spawners and Georges Bank cod in LG12, but no differentiation in LG7 between any of our population samples. This may be due to either our reduced sampling size, or sampling solely from stationary, coastal cod populations.

It is interesting to note that, while these putative supergenes are likely to have been initially protected from recombination by chromosomal inversion [32], the expected result of decreased genetic diversity within inversions is not seen in our data. In fact we see both a larger density of SNPs and a greater proportion of exonic SNPs within the linkage block on LG02. In addition, several unlinked low allele frequency SNPs exist in between the highly linked loci, suggesting that time has allowed mutation to occur within the linkage blocks, as recombination is an unlikely source for the unlinked loci. All of these factors suggest that, if these supergenes arose from chromosomal inversions, they are likely to be older, stable, and adaptive inversion polymorphisms [58, 59].

Caution must be taken in describing potential selection factors within linkage disequilibrium clusters, however, because it is unclear as to whether the entire linkage unit is under selection as a true supergene, or is merely the effect of recent strong selection on one or a few genes with genomic hitchhiking of nearby genes. In the three major LD blocks we described here, the block on LG02 was only one with statistically significant within-cluster enrichment of Gene Ontology (GO) categories, suggesting that the LD blocks on linkage groups 7 and 12 may not have functionally linked genes within them.

\section{Evidence for population structure}

Previous work has shown that genetic differentiation is present within the Gulf of Maine and Georges Bank populations of cod $[34,35]$. Most markers in these comparisons showed no structure, but two loci in both data sets showed significant differentiation (the microsatellite 'Gmo132' and the PanI locus). When Kovach et al. [35] removed these loci from their analysis, no patterns of genetic differentiation were found. Likewise, in our data, both principal component and $\mathrm{F}_{\mathrm{ST}}$ analyses show that divergence between the sampled populations is limited solely to the linkage blocks within LG 2, 7, and 12, and not throughout the genome. This suggests that some level of adaptive divergence may be responsible for the 
amount of differentiation historically seen in these genomic regions, though caution must be employed in interpretations of selection in this study due to a low per-population sampling size.

\section{Conclusions}

Strong neutral genetic differentiation between populations implies very low genetic or demographic exchange between them, and as a result, neutral genetic boundaries in fisheries species have long been used to suggest the existence of different stocks $[60,61]$. The lack of strong neutral differentiation does not necessarily mean strong gene flow between fish stocks, as large effective population sizes can reduce the effect of differential genetic drift [62]. However, if natural selection generates differentiation, local adaptation and minimal gene flow is often the interpretation. This may not be true, however, as demographic exchange between two populations may remain high [22]. For example, a strong cline in allele frequency at a single locus in the mussel Mytilus edulis in southern Long Island Sound is generated by natural selection each summer [63]. However, this genetic divergence does not imply different stocks: the southern population under selection is replenished each spring by strong immigration from the north, and there are few demographic barriers between them. Likewise, allele frequencies of multiple genes in linkage groups LG02, LG07 and LG12 shift over spatial scales of $100 \mathrm{~s}$ of miles or temporal scales of decades in cod in Newfoundland $[28,29]$, implying strong selective changes perhaps due to fishing or environmental shifts. As in the mussel example, such genetic differences do not by themselves imply demographic separation of these populations into separate stocks. They also do not imply that selection is ongoing. The action of strong selection in the past can be visible in current populations if past perturbations of gene flow have not yet been erased by migration.

Previous studies of cod within this system [33] have determined that population structure exists between spawning stocks of cod within the Gulf of Maine. However, these studies relied on markers whose physical locations within the genome were unknown, and often only one or a small number of the markers tested showed genetic divergence $[34,35]$. It may be that these markers, which have been interpreted as evidence of population structure, are located within these linkage disequilibrium blocks and are in fact related to their adaptive divergence.

Our data set was designed to provide high coverage of whole genome sequences for relatively small numbers of individuals. Our analysis shows that large regions of select linkage groups appear to be under adaptive divergence between spawning groups. Furthermore, this genetic divergence does not extend to loci on the same linkage map groups within the Gulf of Maine. Together, these results suggest that extensive study of neutral gene divergence within the Gulf of Maine could be accomplished by choosing markers strictly outside known regions of linked polymorphisms. Furthermore, patterns of nucleotide differentiation among linked gene regions might suggest the way natural selection has acted on these regions, and if it continues to shape population genetics of modern cod.

\section{Additional files}

Additional file 1: List of genes found within LD block of LG02. GFF file containing the gene ID, positions, and gene names of the 294 genes found within this linkage disequilibrium block. (TXT $108 \mathrm{~kb}$ )

Additional file 2: List of genes found within LD block of LG07. GFF file containing the gene ID, positions, and gene names of the 306 genes found within this linkage disequilibrium block. (TXT $116 \mathrm{~kb}$ )

Additional file 3: List of genes found within LD block of LG12. GFF file containing the gene ID, positions, and gene names of the 437 genes found within this linkage disequilibrium block. (TXT $166 \mathrm{~kb}$ )

\section{Abbreviations}

cM: Centimorgans; EST: Expressed sequence tag; GB: Georges Bank; GO: Gene ontology; GoM: Gulf of Maine; LD: Linkage disequilibriuml LG, linkage map group; PCA: Principal component analysis; PCR: Polymerase chain reaction; SNP: Single-nucleotide polymorphism

\section{Acknowledgements}

We would like to thank the following people for their contributions to this work: Bill Hoffman (Massachusetts Division of Marine Fisheries) for the collection of specimens, Joe Mellor for the isolation of DNA and coordinating sequencing, Cindy Lawley (Illumina) for connecting the researchers at Gloucester Marine Genomics Institute and Stanford University, and Nina Therkildsen (Cornell University) for advice and background in cod genetics research.

\section{Funding}

The Massachusetts Division of Marine Fisheries funded the collection and DNA sequencing of samples.

Availability of data and materials

The critical datasets generated and analyzed during the current study are available within the Open Science Framework, at the following URL: http://osf.io/epydk/.

Authors' contributions

DW and CM coordinated the collection of samples and sequencing. BTB and SRP performed all analyses and BTB wrote the final manuscript, with editorial assistance from SRP, CM, and DW. All authors read and approved the final manuscript.

Competing interests

The authors declare that they have no competing interests.

Consent for publication

Not applicable.

Ethics approval

All samples were collected by Bill Hoffman from the Massachusetts Division of Marine Fisheries, in accordance with their internal collection permissions and protocols. Samples consisted of $1 \mathrm{~cm}^{2}$ fin clips, an industry-standard non-lethal form of sampling fish for genetic analysis, and no ethics board approval was sought by the Massachusetts Division of Marine Fisheries.

\section{Publisher's Note}

Springer Nature remains neutral with regard to jurisdictional claims in published maps and institutional affiliations. 


\section{Author details}

'Department of Biology, Hopkins Marine Station, Stanford University, 120 Ocean View Boulevard, Pacific Grove, CA 92950, USA. ²Gloucester Marine Genomics Institute, 55 Blackburn Center, Gloucester, MA 01930, USA.

\section{Received: 30 July 2016 Accepted: 25 March 2017}

\section{Published online: 31 March 2017}

\section{References}

1. Turner TL, Hahn MW, Nuzhdin SV. Genomic islands of speciation in Anopheles gambiae. PLoS Biol. 2005;3:e285.

2. Nosil P, Feder JL. Genomic divergence during speciation: causes and consequences. Philos Trans R Soc B Biol Sci. 2012;367:332-42.

3. Barton NH. Genetic hitchhiking. Philos Trans R Soc Lond B Biol Sci. 2000;355: 1553-62.

4. Nosil P, Funk DJ, Ortiz-Barrientos D. Divergent selection and heterogeneous genomic divergence. Mol Ecol. 2009;18:375-402.

5. Malinsky M, Challis RJ, Tyers AM, Schiffels S, Terai Y, Ngatunga BP, et al. Genomic islands of speciation separate cichlid ecomorphs in an East African crater lake. Science (80-). 2015;350:1493-8.

6. Feder $\lrcorner$, Nosil $P$. The efficacy of divergence hitchhiking in generating genomic islands during ecological speciation. Evolution (N Y). 2010;64:1729-47.

7. Cruickshank TE, Hahn MW. Reanalysis suggests that genomic islands of speciation are due to reduced diversity, not reduced gene flow. Mol Ecol. 2014:23:3133-57.

8. Feder $\mathrm{L}$, Egan SP, Nosil P. The genomics of speciation-with-gene-flow. Trends Genet. 2012;28:342-50.

9. Flaxman SM, Feder JL, Nosil P. Genetic hitchhiking and the dynamic buildup of genomic divergence during speciation with gene flow. Evolution ( $\mathrm{Y}$ ). 2013;67:2577-91.

10. Bird CE, Fernandez-Silva I, Skillings DJ, Toonen RJ. Sympatric speciation in the post "Modern Synthesis" era of evolutionary biology. Evol Biol. 2012;39: $158-80$

11. Butlin RK. Population genomics and speciation. Genetica. 2010;138:409-18

12. Marques DA, Lucek K, Meier Jl, Mwaiko S, Wagner CE, Excoffier L, et al. Genomics of rapid incipient speciation in sympatric threespine stickleback. PLoS Genet. 2016;12:e1005887.

13. Noor M a F, Bennett SM. Islands of speciation or mirages in the desert? Examining the role of restricted recombination in maintaining species. Heredity (Edinb). 2009;103:439-44.

14. Ashburner M, Lemeunier F. Relationships within the melanogaster species subgroup of the Genus Drosophila (Sophophora). I.Inversion polymorphisms in drosophila melanogaster and drosophila simulans. Proc R Soc London B Biol Sci. 1976;193:137-57.

15. Knibb WR. Chromosome inversion polymorphisms in Drosophila melanogaster II. Geographic clines and climatic associations in Australasia, North America and Asia. Genetica. 1982;58:213-21.

16. Kennington WJ, Partridge L, Hoffmann AA. Patterns of diversity and linkage disequilibrium within the cosmopolitan inversion In(3R)Payne in Drosophila melanogaster are indicative of coadaptation. Genetics. 2006;172:1655-63.

17. Umina PA, Weeks AR, Kearney MR, McKechnie SW, Hoffmann AA. A rapid shift in a classic clinal pattern in Drosophila reflecting climate change. Science (80-). 2005;308:691-3.

18. Balanya J, Sole E, Oller JM, Sperlich D, Serra L. Long-term changes in the chromosomal inversion polymorphism of Drosophila subobscura. II European populations. J Zool Syst Evol Res. 2004;42:191-201.

19. Lee $\mathrm{YW}$, Fishman L, Kelly JK, Willis JH. A segregating inversion generates fitness variation in Yellow Monkeyflower (Mimulus guttatus). Genetics. 2016; 202:1473-84.

20. Schwander T, Libbrecht R, Keller L. Supergenes and complex phenotypes. Curr Biol. 2014;24:R288-94.

21. Bay RA, Rose NH, Barrett RDH, Bernatchez L, Ghalambor CK, Lasky JR, et al. Predicting responses to contemporary environmental change using evolutionary response architectures. Am Nat. 2017;189. do:10.1086/691233.

22. Tigano A, Friesen VL. Genomics of local adaptation with gene flow. Mol Ecol. 2016:25:2144-64

23. Hubert S, Higgins B, Borza T, Bowman S. Development of a SNP resource and a genetic linkage map for Atlantic cod (Gadus morhua). BMC Genomics. 2010;11:191.

24. Borza T, Higgins B, Simpson G, Bowman S. Integrating the markers Pan I and haemoglobin with the genetic linkage map of Atlantic cod (Gadus morhua). BMC Res Notes. 2010;3:261-7.
25. Bradbury IR, Hubert S, Higgins B, Bowman S, Borza T, Paterson IG, et al. Genomic islands of divergence and their consequences for the resolution of spatial structure in an exploited marine fish. Evol Appl. 2013;6:450-61.

26. Bradbury IR, Hubert $S$, Higgins B, Borza $T$, Bowman $S$, Paterson IG, et al. Parallel adaptive evolution of Atlantic cod on both sides of the Atlantic Ocean in response to temperature. Proc Biol Sci. 2010;277:3725-34.

27. Hemmer-Hansen J, Nielsen EE, Therkildsen NO, Taylor MI, Ogden R, Geffen $\mathrm{AJ}$, et al. A genomic island linked to ecotype divergence in Atlantic cod. Mol Ecol. 2013;22:2653-67.

28. Therkildsen NO, Hemmer-Hansen J, Als TD, Swain DP, Morgan MJ, Trippel E a, et al. Microevolution in time and space: SNP analysis of historical DNA reveals dynamic signatures of selection in Atlantic cod. Mol Ecol. 2013;22:2424-40.

29. Therkildsen NO, Hemmer-Hansen J, Hedeholm RB, Wisz MS, Pampoulie C, Meldrup D, et al. Spatiotemporal SNP analysis reveals pronounced biocomplexity at the northern range margin of Atlantic cod Gadus morhua. Evol Appl. 2013;6: 690-705.

30. Tørresen OK, Star B, Jentoft S, Reinar WB, Grove H, Miller JR, et al. An improved genome assembly uncovers prolific tandem repeats in Atlantic cod. BMC Genomics. 2017;18:95.

31. Sodeland $M$, Jorde PE, Lien S, Jentoft S, Berg PR, Grove H, et al. "Islands of divergence" in the Atlantic Cod genome represent polymorphic chromosomal rearrangements. Genome Biol Evol. 2016;8:1012-22.

32. Berg PR, Star B, Pampoulie C, Sodeland M, Barth JMI. Three chromosomal rearrangements promote genomic divergence between migratory and stationary ecotypes of Atlantic cod. Sci Rep. 2016;6:23246.

33. Zemeckis DR, Martins D, Kerr LA, Cadrin SX. Stock identification of Atlantic cod (Gadus morhua) in US waters: an interdisciplinary approach. ICES J Mar Sci. 2014:71:1490-506

34. Wirgin I, Kovach Al, Maceda L, Roy NK, Waldman J, Berlinsky DL. Stock identification of Atlantic cod in U.S. waters using microsatellite and single nucleotide polymorphism DNA analyses. Trans Am Fish Soc. 2007;136:375-91.

35. Kovach Al, Breton TS, Berlinsky DL, Maceda L, Wirgin I. Fine-scale spatial and temporal genetic structure of Atlantic cod off the Atlantic coast of the USA. Mar Ecol Prog Ser. 2010:410:177-95.

36. Therkildsen NO, Palumbi SR. Practical low-coverage genome-wide sequencing of hundreds of individually barcoded samples for population and evolutionary genomics in non-model species. Mol Ecol Resour. 2017;17: 194-208.

37. Bolger AM, Lohse M, Usadel B. Trimmomatic: A flexible trimmer for Illumina sequence data. Bioinformatics. 2014;30:1-7.

38. Li H, Handsaker B, Wysoker A, Fennell T, Ruan J, Homer N, et al. The sequence alignment/Map format and SAMtools. Bioinformatics. 2009;25:2078-9.

39. Garrison E, Marth G. Haplotype-based variant detection from short-read sequencing. arXiv Prepr arXiv1207.3907. 2012;9:1.

40. Danecek P, Auton A, Abecasis G, Albers CA, Banks E, DePristo MA, et al. The variant call format and VCFtools. Bioinformatics. 2011;27:2156-

41. Alexa A, Rahnenfuhrer J. topGO: Enrichment Analysis for Gene Ontology. R package version 2.24.0

42. Goudet J. FSTAT, a program to estimate and test gene diversities and fixation indices (version 2.9.3). 2001

43. Weir BS, Cockerham CC. Estimating F-statistics for the analysis of population structure. Evolution (N Y). 1984:38:1358-70.

44. Skotte L, Korneliussen TS, Albrechtsen A. Estimating individual admixture proportions from next generation sequencing data. Genetics. 2013;195: 693-702.

45. Evanno G, Regnaut S, Goudet J. Detecting the number of clusters of individuals using the software STRUCTURE: A simulation study. Mol Ecol. 2005; 14:2611-20.

46. Berg PR, Jentoft S, Star B, Ring KH, Knutsen H, Lien S, et al. Adaptation to low salinity promotes genomic divergence in Atlantic cod (Gadus morhua L.) Genome Biol Evol. 2015:7:1644-63.

47. Sanford E, Kelly MW. Local adaptation in marine invertebrates. Annu Rev Mar Sci. 2011:3:509-35.

48. Whiteley NM, Rastrick SPS, Lunt DH, Rock J. Latitudinal variations in the physiology of marine gammarid amphipods. J Exp Mar Bio Ecol. 2011;400:70-7.

49. Pespeni MH, Garfield DA, Manier MK, Palumbi SR. Genome-wide polymorphisms show unexpected targets of natural selection. Proc R Soc B Biol Sci. 2011;279:1412-20.

50. Pespeni MH, Barney BT, Palumbi SR. Differences in the regulation of growth and biomineralization genes revealed through long-term common-garden 
acclimation and experimental genomics in the Purple Sea Urchin. Evolution (N Y). 2013;67:1901-14.

51. De Wit P, Palumbi SR. Transcriptome-wide polymorphisms of red abalone (Haliotis rufescens) reveal patterns of gene flow and local adaptation. Mol Ecol. 2012;22:2884-97.

52. Bay RA, Palumbi SR. Multilocus adaptation associated with heat resistance in reef-building corals. Curr Biol. 2014;24:2952-6.

53. Richardson JL, Urban MC. Strong selection barriers explain microgeographic adaptation in wild salamander populations. Evolution (N Y). 2013;67:1729-40.

54. True ED, Wiitala SA. Annual temperature curves in twelve regions of the Gulf of Maine. Northwest Atl Fish Organ Sci Counc Stud. 1977;14:21-7.

55. Hutchings JA, Myers RA. Timing of cod reproduction: interannual variability and the influence of temperature. Mar Ecol Prog Ser. 1994;108:21-31.

56. Hochachka PW, Somero GN. Biochemical Adaptation: Mechanism and Process in Physiological Evolution. New York: Oxford University Press; 2002

57. Long Y, Li L, Li Q, He X, Cui Z. Transcriptomic characterization of temperature stress responses in larval zebrafish. PLoS One. 2012;7:e37209.

58. McGaugh SE, Noor MAF. Genomic impacts of chromosomal inversions in parapatric Drosophila species. Philos Trans R Soc Lond B Biol Sci. 2012;367: 422-9.

59. Guerrero RF, Rousset F, Kirkpatrick M. Coalescent patterns for chromosomal inversions in divergent populations. Philos Trans R Soc Lond B Biol Sci. 2012;367:430-8

60. Grant WS, Milner GB, Krasnowski P, Utter FM. Use of biochemical genetic variants for identification of Sockeye salmon (Oncorhynchus nerka) stocks in Cook Inlet, Alaska. Can J Fish Aquat Sci. 1980;37:1236-47.

61. Waples RS, Winans GA, Utter FM, Mahnken C. Genetic approaches to the management of Pacific salmon. Fisheries. 1990;15:19-25.

62. Hauser L, Carvalho GR. Paradigm shifts in marine fisheries genetics: Ugly hypotheses slain by beautiful facts. Fish Fish. 2008;9:333-62.

63. Koehn RK, Newell RIE, Immermann F. Maintenance of an aminopeptidase allele frequency cline by natural selection. Proc Natl Acad Sci U S A. 1980;77: 5385-9.

\section{Submit your next manuscript to BioMed Central and we will help you at every step:}

- We accept pre-submission inquiries

- Our selector tool helps you to find the most relevant journal

- We provide round the clock customer support

- Convenient online submission

- Thorough peer review

- Inclusion in PubMed and all major indexing services

- Maximum visibility for your research

Submit your manuscript at www.biomedcentral.com/submit 